\title{
Fourier-Bessel Expansions with Arbitrary Radial Boundaries
}

\author{
Muhammad A. Mushref \\ P. O. Box 9772, Jeddah, Saudi Arabia \\ E-mail:mmushref@yahoo.co.uk \\ Received January 10, 2010; revised February 21, 2010; accepted February 23, 2010
}

\begin{abstract}
Series expansion of single variable functions is represented in Fourier-Bessel form with unknown coefficients. The proposed series expansions are derived for arbitrary radial boundaries in problems of circular domain. Zeros of the generated transcendental equation and the relationship of orthogonality are employed to find the unknown coefficients. Several numerical and graphical examples are explained and discussed.
\end{abstract}

Keywords: Fourier-Bessel Analysis, Boundary Value Problems, Orthogonality of Bessel Functions

\section{Introduction}

Several boundary value problems in the applied sciences are frequently solved by expansions in cylindrical harmonics with infinite terms. Problems of circular domain with rounded surfaces often generate infinite series of Bessel functions of the first and second types with unknown coefficients. In this case, the intention is to find the series coefficients which should satisfy the boundary conditions.

The subject of Fourier-Bessel series expansions was investigated and examined in many texts [1-10]. Nearly all of them has derived cylindrical harmonics expansions in $J_{0}(r)$ for the interval $[0, a]$ only, where $J_{0}(r)$ is the Bessel function of the first kind with order zero and argument $r$ [8]. The existence of the origin point excludes $Y_{0}(r)$, Bessel function of the second kind with order zero and argument $r$, because it goes to negative infinity as $r$ approaches zero [9]. Both $J_{0}(r)$ and $Y_{0}(r)$ are shown plotted in Figure 1.

In many other problems in the applied sciences, the interval of expansion is found to be $[a, b]$ such that $a, b$ $\in \mathbf{R}$. An example of this could be a hollow cylinder in heat conduction problems or a circular band in vibrations analysis solved in the cylindrical coordinate system. In this case, cylindrical harmonics expansions in both $J_{0}(r)$ and $Y_{0}(r)$ are necessary.

In this paper, the derivation of cylindrical harmonics expansion of a single variable function in $[a, b]$ in both $J_{0}(r)$ and $Y_{0}(r)$ is solved. In accordance with the boundaries at $r=a$ and $r=b$, zeros of the obtained transcendental equation are first calculated. As shown in Figure 2, the solution region is for $a \leq r \leq b$ where the desired series expansions are forced to be zero at $r=a$ and $r=b$ respectively. Unknown coefficients are then found and the complete series expansion can be achieved.

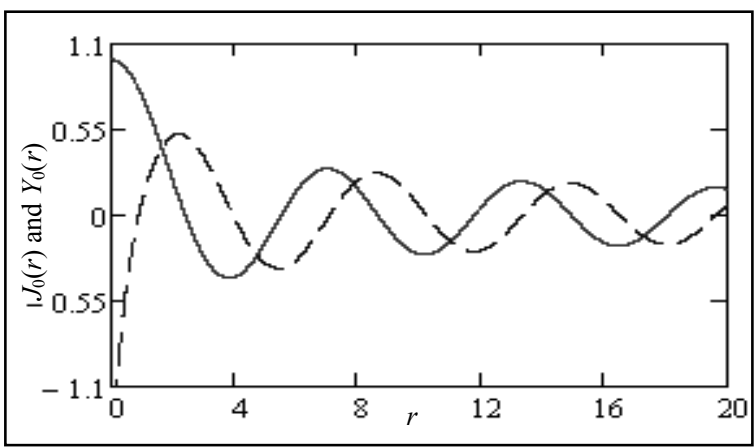

Figure 1. Equation (6), $-J_{0}(r),--Y_{0}(r)$.

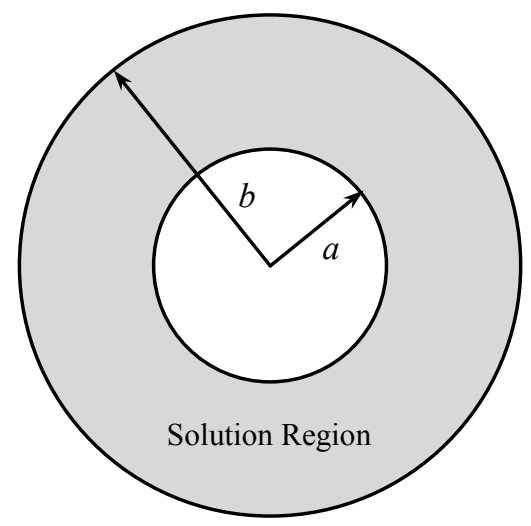

Figure 2. The solution region in radial boundaries. 


\section{Formulation and Solution}

The Bessel differential equation of order zero is well known as $[1,4]$ :

$$
r \frac{d^{2}}{d r^{2}} f(r)+\frac{d}{d r} f(r)+\alpha^{2} r f(r)=0
$$

$\forall \alpha$ and $r \in \mathbf{R}$ and $a \leq r \leq b$.

The general solution to Equation (1) for real values of $\alpha$ is known to be $[2,3]$ :

$$
f(r)=\sum_{n=0}^{\infty} A_{n} J_{0}(\alpha r)+B_{n} Y_{0}(\alpha r)
$$

As in Equation (1), the assumed boundary conditions at $r=a$ and $r=b$ are of Dirichlet type as $f(a)=0$ and $f(b)$ $=0$ respectively. Both $A_{n}$ and $B_{n}$ are then related as:

$$
\begin{aligned}
& A_{n}=-\frac{Y_{0}(\alpha a)}{J_{0}(\alpha a)} B_{n} \\
& A_{n}=-\frac{Y_{0}(\alpha b)}{J_{0}(\alpha b)} B_{n}
\end{aligned}
$$

Going after the elimination method, the transcendental equation can be obtained as:

$$
J_{0}(\alpha a) Y_{0}(\alpha b)-J_{0}(\alpha b) Y_{0}(\alpha a)=0
$$

In order for Equation (5) to be satisfied, there exist many zeros or values of $\alpha$ to be calculated. Thus, in all former and coming equations $\alpha$ can be replaced by $\alpha_{n}$ which are the zeros obtained from the transcendental equation $\forall n \in \mathbf{I}$. That is:

$$
J_{0}\left(\alpha_{n} a\right) Y_{0}\left(\alpha_{n} b\right)-J_{0}\left(\alpha_{n} b\right) Y_{0}\left(\alpha_{n} a\right)=0
$$

The orthogonality feature of Bessel functions can be applied to Equation (2) by multiplying both sides by $r\left[A_{m} J_{0}\left(\alpha_{m} r\right)+B_{m} Y_{0}\left(\alpha_{m} r\right)\right]$ and integrating it over all possible values of $r$ from $a$ to $b$ as:

$$
\sum_{n=0}^{\infty} \int_{a}^{b} r C_{n}(r) C_{m}(r) d r=\int_{a}^{b} r C_{m}(r) f(r) d r
$$

where,

$$
\begin{gathered}
C_{m}(r)=A_{m} J_{0}\left(\alpha_{m} r\right)+B_{m} Y_{0}\left(\alpha_{m} r\right) \\
C_{n}(r)=A_{n} J_{0}\left(\alpha_{n} r\right)+B_{n} Y_{0}\left(\alpha_{n} r\right)
\end{gathered}
$$

The terms under the summation in the left side of Equation (7) are zeros for all values of $m \neq n[5,6,7]$. Hence, Equation (7) can be simplified to:

$$
\int_{a}^{b} r C_{n}(r)\left\{C_{n}(r)-f(r)\right\} d r=0
$$

Either Equation (3) or (4) can help. Using Equation (3) we can obtain the $B_{n}$ coefficients as:

$$
B_{n}=\frac{\int_{a}^{b} r S_{0}\left(\alpha_{n} r\right) f(r) d r}{\int_{a}^{b} r\left[S_{0}\left(\alpha_{n} r\right)\right]^{2} d r}
$$

where, $S_{0}\left(\alpha_{n} r\right)$ is given by:

$$
S_{0}\left(\alpha_{n} r\right)=Y_{0}\left(\alpha_{n} r\right)-\frac{Y_{0}\left(\alpha_{n} a\right)}{J_{0}\left(\alpha_{n} a\right)} J_{0}\left(\alpha_{n} r\right)
$$

By Equation (3) or (4), the $A_{n}$ coefficients can also be found. Once the coefficients $A_{n}$ and $B_{n}$ are calculated, the function $f(r)$ can be expanded as in Equation (2).

\section{Numerical Examples}

The transcendental expression in Equation (6) shows a gradual decay as $\alpha$ increases which mean small magnitudes between high zeros. This leads to the convergence of the series in Equation (2) above as $n$ increases. As a consequence, a finite number of terms in Equation (2) can be sufficient for numerical approximations.

The zeros are first evaluated using the transcendental cross product Bessel functions equation for the interval $[a, b]$. A graph of Equation (6) is shown in Figure 3 for the solution regions $[0.65,2.5]$ and $[0.65,5]$. Table 1 shows the first 50 zeros of Equation (6) for $a=0.65$ and $b=2.5$. Zeros obtained from the transcendental equation changes according to the values of $a$ and $b$ assumed for the solution region. The data presented in Table 1 indicates that the calculated zeros are not periodic and should be calculated using a proper numerical technique.

Let's assume that the function $f(r)$ to be expanded as in Equation (2) is $\sin (r)$ with a radial solution region in $[0.65,2.5]$. The coefficients $B_{n}$ can be evaluated from Equation (11) and the $A_{n}$ coefficients are then obtained by Equation (3). Both coefficients are shown in Tables 2 and 3 respectively for $n=0$ to 49 .

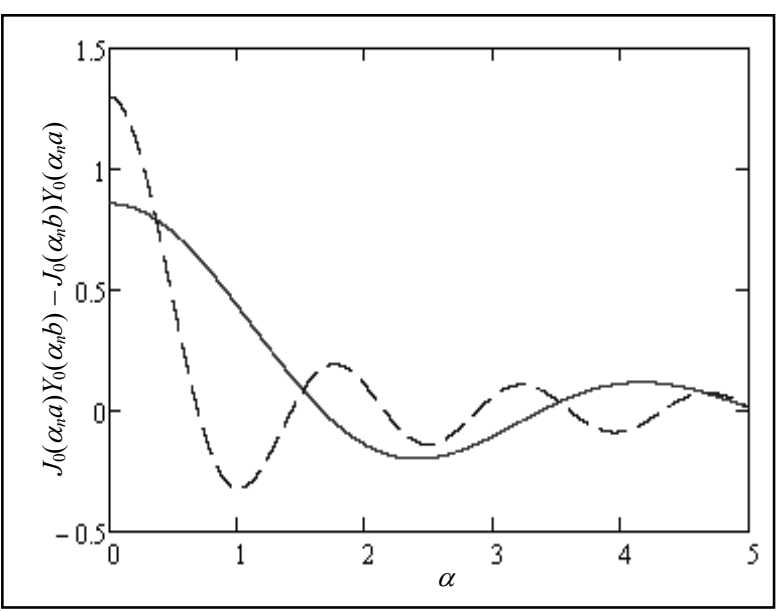

Figure 3. Equation (6), $-[0.65,2.5],--[0.65,5]$. 
Many variations can be noticed for the numerical values of $A_{n}$ and $B_{n}$ with a general absolute scale of $<1$ except for $B_{0}=2.328$. Some coefficients are in the order of $\times 10^{-3}$ meaning that their associated terms are very small such as $B_{4}$ and $A_{31}$ in Tables 2 and 3 respectively.

The function $\sin (r)$ and its approximate expansions are plotted in Figure 4. Summation over the first 10 terms produced an acceptable estimation in the interval [0.65, 2.5] with some apparent oscillations around the exact function. An improved approximate expansion is also plotted for $n=0$ to 49 with less fluctuations in the same radial domain.

Table 1. First fifty zeros of Equation (6) in [0.65, 2.5].

\begin{tabular}{cccccccccc}
\hline$n$ & $\alpha_{n}$ & $n$ & $\alpha_{n}$ & $n$ & $\alpha_{n}$ & $n$ & $\alpha_{n}$ & $n$ & $\alpha_{n}$ \\
\hline 0 & 1.663 & 10 & 18.676 & 20 & 35.659 & 30 & 52.642 & 40 & 69.624 \\
1 & 3.376 & 11 & 20.374 & 21 & 37.358 & 31 & 54.34 & 41 & 71.322 \\
2 & 5.08 & 12 & 22.073 & 22 & 39.056 & 32 & 56.038 & 42 & 73.02 \\
3 & 6.782 & 13 & 23.771 & 23 & 40.754 & 33 & 57.736 & 43 & 74.718 \\
4 & 8.4815 & 14 & 25.47 & 24 & 42.452 & 34 & 59.434 & 44 & 76.416 \\
5 & 10.182 & 15 & 27.168 & 25 & 44.151 & 35 & 61.133 & 45 & 78.115 \\
6 & 11.881 & 16 & 28.866 & 26 & 45.849 & 36 & 62.831 & 46 & 79.813 \\
7 & 13.579 & 17 & 30.564 & 27 & 47.547 & 37 & 64.529 & 47 & 81.511 \\
8 & 15.279 & 18 & 32.263 & 28 & 49.245 & 38 & 66.227 & 48 & 83.209 \\
9 & 16.977 & 19 & 33.961 & 29 & 50.943 & 39 & 67.925 & 49 & 84.907 \\
\hline
\end{tabular}

Table 2. First fifty $B_{n}$ for $f(r)=\sin (r)$ in $[0.65,2.5]$.

\begin{tabular}{cccccccccc}
\hline$n$ & $B_{n}$ & $n$ & $B_{n}$ & $n$ & $B_{n}$ & $n$ & $B_{n}$ & $n$ & $B_{n}$ \\
\hline 0 & 2.328 & 10 & 0.154 & 20 & -0.300 & 30 & -0.114 & 40 & 0.206 \\
1 & -0.101 & 11 & -0.138 & 21 & $7.1 \mathrm{E}-3$ & 31 & 0.084 & 41 & $1.3 \mathrm{E}-3$ \\
2 & -0.703 & 12 & 0.228 & 22 & 0.267 & 32 & -0.123 & 42 & -0.205 \\
3 & 0.234 & 13 & 0.064 & 23 & -0.082 & 33 & -0.047 & 43 & 0.057 \\
4 & $-4.8 \mathrm{E}-3$ & 14 & -0.385 & 24 & -0.030 & 34 & 0.250 & 44 & 0.042 \\
5 & -0.181 & 15 & 0.048 & 25 & 0.087 & 35 & -0.025 & 45 & -0.068 \\
6 & 0.455 & 16 & 0.231 & 26 & -0.212 & 36 & -0.173 & 46 & 0.148 \\
7 & 0.030 & 17 & -0.110 & 27 & -0.024 & 37 & 0.074 & 47 & 0.024 \\
8 & -0.478 & 18 & 0.082 & 28 & 0.272 & 38 & -0.036 & 48 & -0.212 \\
9 & 0.105 & 19 & 0.081 & 29 & -0.054 & 39 & -0.061 & 49 & 0.037 \\
\hline
\end{tabular}

Table 3. First fifty $A_{n}$ for $f(r)=\sin (r)$ in $[0.65,2.5]$.

\begin{tabular}{cccccccccc}
\hline$n$ & $A_{n}$ & $n$ & $A_{n}$ & $n$ & $A_{n}$ & $n$ & $A_{n}$ & $n$ & $A_{n}$ \\
\hline 0 & -0.475 & 10 & 0.424 & 20 & 0.126 & 30 & -0.242 & 40 & -0.109 \\
1 & 0.462 & 11 & -0.016 & 21 & -0.102 & 31 & $2.1 \mathrm{E}-3$ & 41 & 0.074 \\
2 & -0.547 & 12 & -0.346 & 22 & 0.159 & 32 & 0.229 & 42 & -0.099 \\
3 & -0.114 & 13 & 0.111 & 23 & 0.052 & 33 & -0.067 & 43 & -0.044 \\
4 & 0.675 & 14 & 0.021 & 24 & -0.297 & 34 & -0.036 & 44 & 0.218 \\
5 & -0.092 & 15 & -0.110 & 25 & 0.034 & 35 & 0.075 & 45 & -0.019 \\
6 & -0.338 & 16 & 0.279 & 26 & 0.193 & 36 & -0.174 & 46 & -0.160 \\
7 & 0.170 & 17 & 0.025 & 27 & -0.087 & 37 & -0.024 & 47 & 0.064 \\
8 & -0.143 & 18 & -0.333 & 28 & 0.054 & 38 & 0.236 & 48 & -0.023 \\
9 & -0.111 & 19 & 0.070 & 29 & 0.069 & 39 & -0.044 & 49 & -0.057 \\
\hline
\end{tabular}

Table 4. First fifty $B_{n}$ for $f(r)=\cos (r)$ in $[0.65,2.5]$.

\begin{tabular}{cccccccccc}
\hline$n$ & $B_{n}$ & $n$ & $B_{n}$ & $n$ & $B_{n}$ & $n$ & $B_{n}$ & $n$ & $B_{n}$ \\
\hline 0 & -0.129 & 10 & -0.067 & 20 & 0.131 & 30 & 0.050 & 40 & -0.090 \\
1 & 0.338 & 11 & 0.571 & 21 & -0.030 & 31 & -0.351 & 41 & $-5.5 \mathrm{E}-3$ \\
2 & 0.286 & 12 & -0.099 & 22 & -0.116 & 32 & 0.053 & 42 & 0.089 \\
3 & -0.919 & 13 & -0.264 & 23 & 0.342 & 33 & 0.195 & 43 & -0.237 \\
4 & $2.0 \mathrm{E}-3$ & 14 & 0.167 & 24 & 0.013 & 34 & -0.109 & 44 & -0.018 \\
5 & 0.732 & 15 & -0.199 & 25 & -0.364 & 35 & 0.105 & 45 & 0.281 \\
6 & -0.196 & 16 & -0.100 & 26 & 0.092 & 36 & 0.075 & 46 & -0.064 \\
7 & -0.122 & 17 & 0.457 & 27 & 0.100 & 37 & -0.306 & 47 & -0.100 \\
8 & 0.207 & 18 & -0.036 & 28 & -0.118 & 38 & 0.016 & 48 & 0.092 \\
9 & -0.433 & 19 & -0.338 & 29 & 0.223 & 39 & 0.256 & 49 & -0.153 \\
\hline
\end{tabular}

In addition, $f(r)=\cos (r)$ is expanded as in Equation (2) and the first fifty coefficients are listed in Tables $\mathbf{4}$ and $\mathbf{5}$ for the $B_{n}$ and $A_{n}$ respectively. Similar to the $\sin (r)$, the $\cos (r)$ coefficients go through several variations with a general absolute scale of $<1$ except $A_{1}=-1.550$. Also, only four coefficients are in the order of $\times 10^{-3}$ implying that their related terms in the series are extremely small such as $B_{4}$ and $A_{41}$ in Tables 4 and 5 respectively. 
Table 5. First fifty $A_{n}$ for $f(r)=\cos (r)$ in $[0.65,2.5]$.

\begin{tabular}{|c|c|c|c|c|c|c|c|c|c|}
\hline$n$ & $A_{n}$ & $n$ & $A_{n}$ & $n$ & $A_{n}$ & $n$ & $A_{n}$ & $n$ & $A_{n}$ \\
\hline 0 & 0.026 & 10 & -0.184 & 20 & -0.055 & 30 & 0.105 & 40 & 0.047 \\
\hline 2 & 0.222 & 12 & 0.150 & 22 & -0.069 & 32 & -0.100 & 42 & 0.043 \\
\hline 4 & -0.287 & 14 & $-9.1 \mathrm{E}-3$ & 24 & 0.129 & 34 & 0.016 & 44 & -0.095 \\
\hline 5 & 0.371 & 15 & 0.455 & 25 & -0.139 & 35 & -0.314 & 45 & 0.080 \\
\hline 6 & 0.145 & 16 & -0.121 & 26 & -0.084 & 36 & 0.076 & 46 & 0.070 \\
\hline 9 & 0.458 & 19 & -0.289 & 29 & -0.286 & 39 & 0.182 & 49 & 0.235 \\
\hline
\end{tabular}

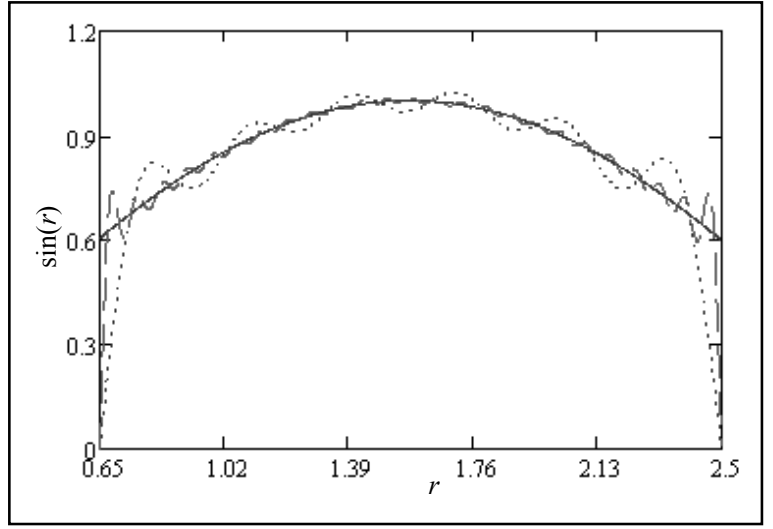

Figure 4. $\sin (r), \cdots$ Equation (2) with $n=0$ to 10, - Equation (2) with $n=0$ to 49.

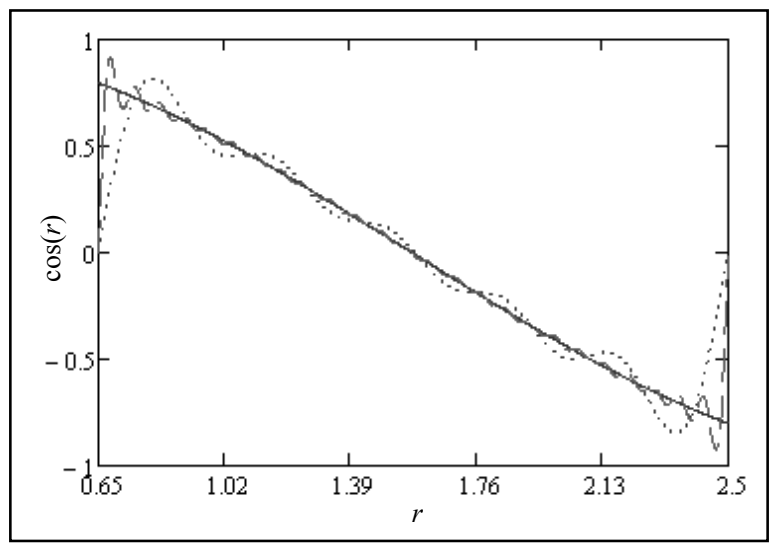

Figure 5. - $\cos (r), \cdots$ Equation (2) with $n=0$ to 10, - Equation (2) with $n=0$ to 49.

The function $\cos (r)$ and its estimated expansions are shown plotted in Figure 5. Finite summation over the first 10 terms generated a satisfactory estimation in the interval $[0.65,2.5]$ with several obvious oscillations close to the exact function. A better approximate expansion is also plotted for $n=0$ to 49 with less fluctuations in the same solution region.

The calculated coefficients for the function $e^{r}$ are also shown in Tables 6 and 7 for $B_{n}$ and $A_{n}$ respectively. Apparently, the coefficients swing around the exact values with an absolute level of $>1$ or $<1$.

The greatest values in Tables 6 and 7 are found as $B_{0}$ $=13.852$ and $A_{1}=11.499$. In addition, no coefficients are calculated in the order of $\times 10^{-3}$ implying that all coefficients are to be included in the series expansion.

The function $\exp (r)$ and its estimated expansions are shown plotted in Figure 6 in $[0.65,2.5]$. A satisfactory estimation of a finite summation over the first 10 terms are generated with several oscillations close to the exact function. A good approximated expansion is also plotted for $n=0$ to 49 with fewer variations in the same solution region.

The last numerical example to be discussed is the square function expressed as:

$$
f(r)=\left\{\begin{array}{cc}
1 & 1.26 \leq r \leq 1.88 \\
-1 & \text { otherwise }
\end{array}\right.
$$

The calculated $B_{n}$ and $A_{n}$ coefficients for this function are shown in Tables 8 and 9 respectively. Similar to former expansions, both coefficients vary about the exact values of Equation (13). The $B_{n}$ coefficients have a general absolute level of $<1$ except $B_{2}, B_{8}, B_{14}, B_{20}$ and $B_{26}$ that have an absolute scale of $>1$. Furthermore, the $A_{n}$ coefficients show an absolute level of $<1$ except the absolute values of $A_{2}, A_{32}, A_{38}$ and $A_{44}$ that are $>1$. Some $B_{n}$ and $A_{n}$ coefficients are calculated in the order of $\times 10^{-3}$ like $A_{0}$ or

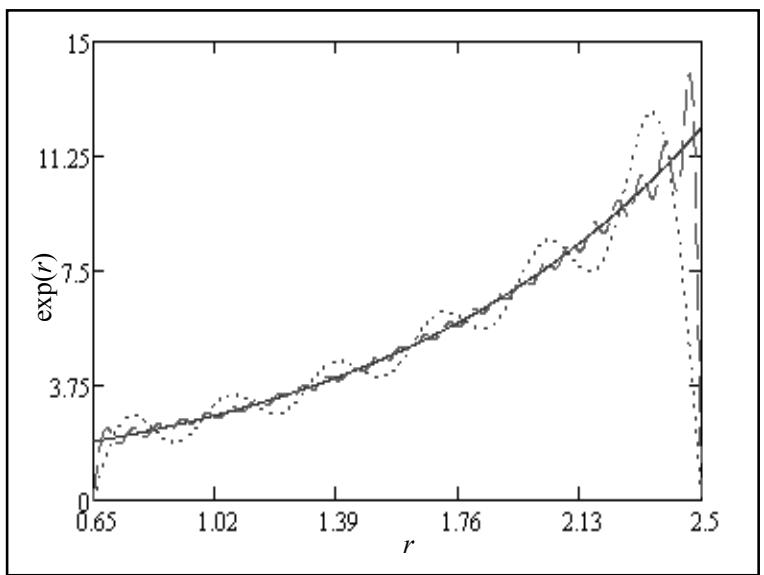

Figure 6. - $\exp (r), \cdots$ Equation (2) with $n=0$ to 10, - Equation (2) with $n=0$ to 49. 
Table 6. First fifty $B_{n}$ for $f(r)=\exp (r)$ in $[0.65,2.5]$.

\begin{tabular}{cccccccccc}
\hline$n$ & $B_{n}$ & $N$ & $B_{n}$ & $n$ & $B_{n}$ & $n$ & $B_{n}$ & $n$ & $B_{n}$ \\
\hline 0 & 13.852 & 10 & 2.217 & 20 & -4.350 & 30 & -1.660 & 40 & 2.987 \\
1 & -2.506 & 11 & -5.266 & 21 & 0.274 & 31 & 3.254 & 41 & 0.051 \\
2 & -9.361 & 12 & 3.298 & 22 & 3.873 & 32 & -1.780 & 42 & -2.971 \\
3 & 8.069 & 13 & 2.443 & 23 & -3.169 & 33 & -1.813 & 43 & 2.202 \\
4 & -0.068 & 14 & -5.566 & 24 & -0.433 & 34 & 3.618 & 44 & 0.602 \\
5 & -6.632 & 15 & 1.841 & 25 & 3.372 & 35 & -0.971 & 45 & -2.611 \\
6 & 6.506 & 16 & 3.343 & 26 & -3.078 & 36 & -2.505 & 46 & 2.140 \\
7 & 1.113 & 17 & -4.227 & 27 & -0.931 & 37 & 2.841 & 47 & 0.932 \\
8 & -6.867 & 18 & 1.182 & 28 & 3.937 & 38 & -0.525 & 48 & -3.072 \\
9 & 3.985 & 19 & 3.127 & 29 & -2.069 & 39 & -2.371 & 49 & 1.419 \\
\hline
\end{tabular}

Table 7. First fifty $A_{n}$ for $f(r)=\exp (r)$ in $[0.65,2.5]$.

\begin{tabular}{ccccccccccc}
\hline$n$ & $A_{n}$ & $n$ & $A_{n}$ & $n$ & $A_{n}$ & $n$ & $A_{n}$ & $n$ & $A_{n}$ \\
\hline 0 & -2.828 & 10 & 6.108 & 20 & 1.821 & 30 & -3.511 & 40 & -1.575 \\
1 & 11.499 & 11 & -0.625 & 21 & -3.915 & 31 & 0.083 & 41 & 2.841 \\
2 & -7.286 & 12 & -4.992 & 22 & 2.304 & 32 & 3.317 & 42 & -1.431 \\
3 & -3.934 & 13 & 4.263 & 23 & 2.019 & 33 & -2.586 & 43 & -1.691 \\
4 & 9.496 & 14 & 0.304 & 24 & -4.301 & 34 & -0.526 & 44 & 3.168 & -0.747 \\
5 & -3.360 & 15 & -4.213 & 25 & 1.292 & 35 & 2.912 & 45 & -2.318 \\
6 & -4.824 & 16 & 4.033 & 26 & 2.799 & 36 & -2.523 & 46 & 2.490 \\
7 & 6.376 & 17 & 0.969 & 27 & -3.353 & 37 & -0.925 & 47 & -0.336 \\
8 & -2.059 & 18 & -4.814 & 28 & 0.782 & 38 & 3.423 & 48 & -2.185 \\
9 & -4.216 & 19 & 2.675 & 29 & 2.650 & 39 & -1.690 & 49 & -29 \\
\hline
\end{tabular}

Table 8. First fifty $B_{n}$ for Equation (13) in $[0.65,2.5]$.

\begin{tabular}{ccccccccccc}
\hline$n$ & $B_{n}$ & $n$ & $B_{n}$ & $n$ & $B_{n}$ & $n$ & $B_{n}$ & $n$ & $B_{n}$ \\
\hline 0 & 0.026 & 10 & $1.64 \mathrm{E}-3$ & 20 & 1.527 & 30 & -0.021 & 40 & -0.051 \\
1 & 0.1 & 11 & 0.081 & 21 & $-5 \mathrm{E}-3$ & 31 & -0.404 & 41 & $-3 \mathrm{E}-4$ \\
2 & 3.515 & 12 & 0.031 & 22 & -0.025 & 32 & 0.614 & 42 & -0.037 \\
3 & -0.516 & 13 & -0.205 & 23 & 0.042 & 33 & $-9 \mathrm{E}-3$ & 43 & -0.329 \\
4 & $-3 \mathrm{E}-4$ & 14 & 1.968 & 24 & $-5 \mathrm{E}-3$ & 34 & -0.05 & 44 & -0.201 \\
5 & 0.105 & 15 & -0.053 & 25 & -0.37 & 35 & $8 \mathrm{E}-3$ & 45 & -0.07 \\
6 & 0.048 & 16 & $-9.1 \mathrm{E}-3$ & 26 & 1.072 & 36 & -0.033 & 46 & -0.048 \\
7 & -0.076 & 17 & 0.061 & 27 & $6 \mathrm{E}-3$ & 37 & -0.388 & 47 & $-3 \mathrm{E}-3$ \\
8 & 2.447 & 18 & 0.013 & 28 & -0.037 & 38 & 0.179 & 48 & -0.037 \\
9 & -0.17 & 19 & -0.305 & 29 & 0.025 & 39 & -0.039 & 49 & -0.228 \\
\hline
\end{tabular}

Table 9. First fifty $A_{n}$ for Equation (13) in $[0.65,2.5]$.

\begin{tabular}{cccccccccc}
\hline$n$ & $A_{n}$ & $n$ & $A_{n}$ & $n$ & $A_{n}$ & $n$ & $A_{n}$ & $n$ & $A_{n}$ \\
\hline 0 & $-5 \mathrm{E}-3$ & 10 & $4.5 \mathrm{E}-3$ & 20 & -0.639 & 30 & -0.045 & 40 & 0.027 \\
1 & -0.457 & 11 & $9.6 \mathrm{E}-3$ & 21 & 0.069 & 31 & -0.01 & 41 & -0.016 \\
2 & 2.735 & 12 & -0.047 & 22 & -0.015 & 32 & -1.144 & 42 & -0.018 \\
3 & 0.252 & 13 & -0.375 & 23 & -0.027 & 33 & -0.013 & 43 & 0.253 \\
4 & 0.039 & 14 & -0.108 & 24 & -0.052 & 34 & $7.2 \mathrm{E}-3$ & 44 & -1.056 \\
5 & 0.053 & 15 & 0.121 & 25 & -0.142 & 35 & -0.024 & 45 & -0.02 \\
6 & -0.035 & 16 & -0.011 & 26 & -0.975 & 36 & -0.033 & 46 & 0.052 \\
7 & -0.433 & 17 & -0.014 & 27 & 0.02 & 37 & 0.126 & 47 & $-6.8 \mathrm{E}-3$ \\
8 & 0.734 & 18 & -0.053 & 28 & $-7.3 \mathrm{E}-3$ & 38 & -1.168 & 48 & $-4 \mathrm{E}-3$ \\
9 & 0.18 & 19 & -0.261 & 29 & -0.032 & 39 & -0.027 & 49 & 0.352 \\
\hline
\end{tabular}

in the order of $\times 10^{-4}$ such as $B_{41}$ indicating that their associated terms in the series are very small.

The function expressed by Equation (13) and its approximate expansions are plotted in Figure 7. Summation over the first 10 terms produced an acceptable estimation in the interval $[0.65,2.5]$ with some noticeable oscillations around the exact function. A better approximate expansion is also plotted for $n=0$ to 49 with less fluctuations in the same radial domain.

In all graphical plots previously shown, the curves re- turn to zero at the assumed boundaries $a=0.65$ and $b=$ 2.5. In addition, accuracy of the expanded curves may appear better as $n$ increases due to larger number of terms involved in the series and less fluctuations seen around the exact values.

\section{Conclusions}

Functions were expanded as a Fourier-Bessel series summation in both $J_{0}(r)$ and $Y_{0}(r)$. A finite series expan- 


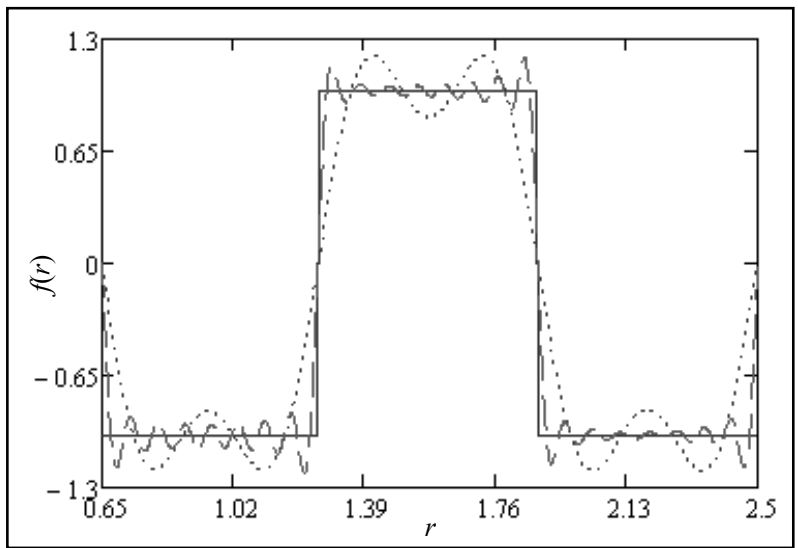

Figure 7. Equation (13), $\cdots$ Equation (2) with $n=0$ to $10,-$ Equation (2) with $n=0$ to 49.

sion was obtained for arbitrary radial boundaries in $[a, b]$. Coefficients were found by calculating the zeros of the transcendental equation and by employing the relationship of orthogonality. A number of examples were numerically and graphically discussed.

\section{References}

[1] G. N. Watson, "A Treatise on the Theory of Bessel Func- tions," Cambridge University Press, United Kingdom, 1996.

[2] H. F. Davis, "Fourier Series and Orthogonal Functions," Dover Publications, Inc., New York, 1989.

[3] W. E. Byerly, "An Elementary Treatise on Fourier's Series," Dover Publications, Inc., New York, 2003.

[4] F. Bowman, "Introduction to Bessel Functions," Dover Publications, Inc., New York, 1958.

[5] N. N. Lebedev, "Special Functions and Their Applications," Dover Publications, Inc., New York, 1972.

[6] E. A. Gonzalez-Velasco, "Fourier Analysis and Boundary Value Problems," Academic Press, San Diego, 1995.

[7] A. Broman, "Introduction to Partial Differential Equations: From Fourier Series to Boundary Value Problems," Dover Publications, Inc., New York, 1970.

[8] P. V. O’Neil, “Advanced Engineering Mathematics,” Wadsworth Publishing Company, California, 1987.

[9] H. Sagan, "Boundary and Eigenvalue Problems in Mathematical Physics," Dover Publications, Inc., New York, 1989.

[10] N. N. Lebedev, I. P. Skalskaya and Y. S. Uflyand, "Worked Problems in Applied Mathematics," Dover Publications, Inc., New York, 1979. 